\title{
PROTOCOLO PARA LA VIGILANCIA DE LA SALUD DEL PROFESORADO CON ATENCIÓN A LA ENFERMEDAD PROFESIONAL
}

\author{
ANTONIO RANCHAL SÁNCHEZ*, \\ MANUEL VAQUERO ABELLÁN**
}

(*) Médico Especialista en Medicina del Trabajo.

Profesor Asociado de la Universidad de Córdoba.

(**) Médico Especialista en Medicina del Trabajo.

Director General de Prevención y Protección Ambiental de la Universidad de Córdoba.

\section{RESUMEN}

El docente es uno de los principales sectores laborales cuantitativa y cualitativamente, siendo esencial un nivel óptimo de salud laboral para realizar adecuadamente su tarea. Desde la entrada en vigor del Real Decreto 1299/2006, los "Nódulos de las cuerdas vocales a causa de los esfuerzos sostenidos de la voz por motivos profesionales" constituyen una enfermedad profesional en el profesorado, cuestión novedosa para este colectivo.

El objetivo de este trabajo es la propuesta de un protocolo para la vigilancia de la salud del profesorado. Cuestión que surge tras realizar un estudio epidemiológico para conocer la forma de enfermar del profesorado de secundaria, a partir de una muestra representativa, considerando variables de salud laboral. Analizamos la patología más frecuente que aparece en la población encuestada, entre la que figura los trastornos de la voz, incluidos los nódulos de las cuerdas vocales. La importancia de diagnosticar esta enfermedad obliga a tenerla en cuenta en los reconocimientos médicos para la Vigilancia de la Salud realizados en el profesorado. De ahí que propongamos un protocolo para facilitar el examen de salud específico, así como una secuencia de actuación ante su posible diagnóstico.

\section{PALABRAS CLAVES}

Vigilancia de la salud, Riesgos ocupacionales en Docentes, Enfermedad profesional, Nódulos de cuerdas vocales.

\begin{abstract}
Teachering is one of the quantitatively and qualitatively main economical sectors, being essential an optimum level of occupational health to adequately perform their task. Since the entry into force of Real Decreto 1299/2006, "Nodules of the vocal cords because of the sustained efforts of the voice for professional reasons" constitute an occupational disease for teachers, a new issue for this group.

The purpose of this paper is the proposal of a procedure for monitoring the health of the teaching staff. This is a matter that arises from an epidemiological research in order to find out how a representative sample of secondary education teaching staff could fall ill, considering variables of occupational health. We have analyzed the most frequent pathology appearing in the surveyed population, among which disorders of the voice are found including vocal cord nodules. The importance of diagnosing this disease requires it to be included in medical check-ups done to the teaching staff for the Health Surveillance. Hence, we propose a procedure to facilitate the specific health exam, as well as a following approach faced with its possible diagnosis.
\end{abstract}

\section{KEY WORDS}

Health Surveillance, Occupational risks and hazards in teaching, Occupational disease, Vocal cord nodules. 


\section{INTRODUCCIÓN}

El docente representa, además del sanitario, uno de los principales colectivos laborales, tanto en número como en función social. Respecto al número, los datos obtenidos del censo muestran ser el grupo más numeroso en la Administración Pública. En cuanto a la función social, pensemos en la trascendencia que implica la formación y educación de las nuevas generaciones. De ahí que resulte esencial un estado adecuado de su salud para el desarrollo de las labores que la sociedad le tiene encomendadas. El informe del Consejo Escolar del Estado del año 2000 reflejaba el "aumento del absentismo laboral, especialmente en el segundo trimestre del curso, con graves repercusiones en el rendimiento de los alumnos" (1).

Un estudio realizado por la Consejería de educación de la Junta de Andalucía sobre una muestra de 122.000 licencias por enfermedad de personal funcionario docente durante el período 1999-2004, indica que las principales enfermedades que afectan al profesorado son: las que afectan a vías respiratorias altas y los trastornos de la voz, los trastornos osteomusculares y las alteraciones de la salud mental, en este orden (2). La mayoría de las investigaciones sobre la incidencia de las enfermedades en el profesorado citan también a estos procesos entre los más frecuentes $(3,4,5)$.

Desde la publicación del Real Decreto 1299/2006 (6) existe una enfermedad profesional para el profesorado: los nódulos de las cuerdas vocales a causa de los esfuerzos sostenidos de la voz por motivos profesionales. Esto hace que deba prestarse una atención especial a los trastornos de la voz en la vigilancia de la salud del profesorado. Fundamentalmente para evitar llegar a esta situación. También para actuar debidamente llegado el caso. La Ley de Prevención de Riesgos Laborales (7) establece que la Vigilancia de la salud ha de ser periódica, específica según los riesgos y con el consentimiento de la persona a quien se realiza el reconocimiento médico. Con objeto de facilitar el desarrollo de la Vigilancia de la salud en el personal docente, proponemos un protocolo para adaptarlo al entorno específico, así como de un cronograma de actuación.

\section{MATERIAL Y MÉTODOS}

\section{Población de Estudio}

Para el estudio epidemiológico previo, la población de referencia fueron profesionales docentes en activo de la provincia de Córdoba, referido al nivel de enseñanza secundaria durante el curso escolar 2004/2005. Se determinó el tamaño de la muestra, realizando una simulación para predeterminar el tamaño de la misma, a partir de una prevalencia esperada del $25 \%$, precisión del $4.5 \%$ y $\mathrm{p}<0.05$. El número mínimo de sujetos adecuado de la muestra estimada fue de 356. La muestra con la que realizamos el estudio ha sido de 361 profesores.

\section{Método Estadístico}

Realizamos un análisis univariante, estimando para las variables cualitativas sus porcentajes y error estándar; $\mathrm{y}$, para las cuantitativas la media, mediana y moda, así como el rango (valores mínimo y máximo), desviación típica, varianza y error estándar de la media. Las representaciones gráficas que utilizamos son: el diagrama de sectores para las variables cualitativas; el diagrama de barras para las cuantitativas discretas, y el histograma y las cajas y bigotes -boxplot- para las cuantitativas continuas.

\section{Soporte Informático}

Se utilizaron los programas EpiInfo 6.04d (2001) para la simulación -determinación del tamaño muestral y el programa EpiInfo 3.01 (2005); con este último se creó una base de datos y, posteriormente se analizaron con este el mismo soporte informático desde el punto de vista estadístico y epidemiológico.

\section{Propuesta de un cuestionario para evaluar la fun- ción fonatoria en profesionales de la docencia}

Para la confección del protocolo se estudiaron y analizaron los protocolos de vigilancia de la salud existentes hasta la fecha, tomando como modelo el de Pantallas de Visualización de Datos.

El cuestionario que planteamos trata de facilitar la obtención de información a partir de la anamnesis, que, junto con la exploración física, el control biológico y los estudios complementarios, conforman la historia clínico-laboral, en función de los riesgos característicos del puesto de trabajo. Está pensado para aplicarlo en los exámenes de salud que realiza el facultativo en medicina del trabajo para la vigilancia de la salud del profesorado de los niveles de enseñanza no universitaria.

El profesorado realiza normalmente las siguientes actividades en su puesto de trabajo: realizar programaciones, preparar clases e impartirlas (teóricas y prácticas), evaluar al alumnado, tutorizarlo y orientarlo, planificar y asistir a reuniones, redactar y 
gestionar documentos (didácticos, curriculares, organizativos...), atención al alumnado durante el periodo lectivo, vigilancia del alumnado durante el recreo, atención a padres, madres, o tutores del alumnado, organización de actividades extraescolares, etc. Realiza la mayor parte de su actividad en el aula, con el grupo de alumnos, por lo que la mayor parte de su jornada laboral debe utilizar la voz, forzándola en numerosas ocasiones. Hecho que varía según la disciplina (caso, por ejemplo el profesorado de educación física). La voz es la principal herramienta del docente para la mayoría de estas tareas.

\section{RESULTADOS}

Mostramos algunos de los datos obtenidos en el estudio epidemiológico previo.

\section{Datos sociodemográficos}

De los 361 profesores, 187 eran mujeres (51.8\%) y 174 hombres, (48.2\%) (Figura 1).

Figura 1. Distribución de la variable género en la muestra de estudio.



Por otra parte, la edad media de los participantes fue de 43,4 años, con un rango de 23 a 64 años. El Intervalo de Confianza (IC) para la edad fue 43,4 \pm $0,8$ años $(\mathrm{p}<0,05)$, (figura 2$)$.

Destacamos de estas variables, la experiencia profesional. Obtuvimos una media de 17,25 años de dedicación. En nuestro estudio, esta experiencia viene determinada, entre otros factores, por los años impartidos de docencia. Dicha variable obtuvo el siguiente IC: $17,25 \pm 0,83$ años, con un rango de 0 41, (figura 3).
Figura 2. Distribución de la variable género en la muestra de estudio.



Figura 3. Representación de la variable antigüedad como docente.



\section{Salud laboral}

Queríamos saber qué tipos de trastornos y con qué frecuencia la padecían o la habían padecido, dentro de los que se citan como más frecuencia en la enseñanza.

\subsection{Patología más frecuente}

La figura 4 muestra la patología más frecuente en la población docente encuestada, agrupada en tres apartados: Osteomuscular, Otorrinolaringológica (ORL) e Infecciones, y Psiquiatría. Se observa como la patología de ORL e Infecciones obtiene mayores tasas de incidencia tanto "de vez en cuando" con el $62,2 \%$, como "frecuentemente" con un $24,2 \%$. Globalmente, obtiene también la mayor tasa $(\mathrm{p}<0,05)$, ya que del total de trabajadores analizados, el $86,4 \%$ presentaron alguna enfermedad perteneciente a ORL 
e infecciones. En segundo lugar, se presentan las patologías osteomusculares con una incidencia del $68,2 \%$, y finalmente las de origen psiquiátrico con una tasa del 53,9\%.

\section{Figura 4. Frecuencia de aparición de patología más frecuente en la muestra.}

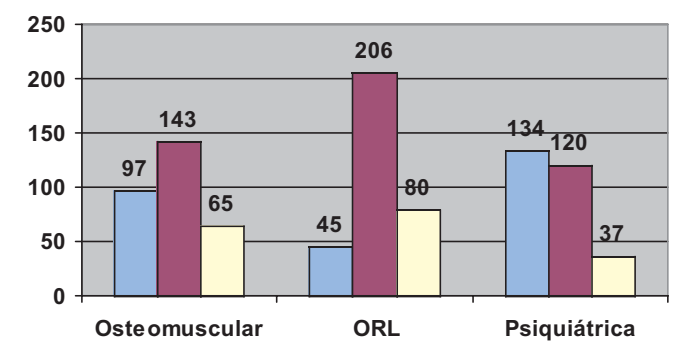

$\square$ Nunca $\square$ De vez en cuando $\square$ Frecuentemente

Datos absolutos expresados en tanto por uno.

Clasificado por síntomas y/o enfermedades (algunos de los encuestados confundían estos términos en sus respuestas), obteníamos los datos siguientes:

- Osteomuscular: Lumbalgia (48,3\%), cervicodorsalgia (31\%).

- ORL e Infecciones: Faringitis (58,5\%), disfonías $(19,5 \%)$.

- Psiquiátricas: Estrés (44,4\%), depresión $25,9 \%)$.

\subsection{Trastornos de la voz}

Queríamos estimar el porcentaje de docentes que padecen habitualmente trastornos de la voz en la población estudiada. Fundamentalmente, porque cuando pasamos la encuesta aparecía ya una patología específica para el personal docente ("nódulos de las cuerdas vocales") en el borrador del nuevo cuadro de enfermedades profesionales. En este sentido, el $32 \%$ de los profesores/as encuestados/as (117 docentes) decían padecer trastornos de la voz (afonías, disfonías...), mientras que 76 trabajadores/as $(40 \%)$ refirieron haber sido diagnosticados/as de alguna enfermedad o lesión relacionada con las cuerdas vocales (nódulos, pólipos, edema, granuloma u otros.

\subsection{Incapacidad temporal}

En cuanto a la duración por incapacidad temporal, la media de días de baja por cada episodio o enfermedad reconocida fue de 40,6 \pm 21 días (IC $\mathrm{p}<0,05)$, y un rango de $1-300$. El $25 \%$ de las incapacidades temporales estaban motivadas, según los docentes encuestados, por trastornos de la voz.

\subsection{Vigilancia de la Salud}

Queríamos saber también si el profesorado participaba habitualmente en los reconocimientos médicos realizados por el Centro de Prevención de Riesgos Laborales (CPRL) de la Consejería de Empleo, que es el Servicio de Prevención propio del personal Funcionario de la Junta de Andalucía, en virtud de la legislación vigente.

Los resultados mostraron que la Vigilancia de la salud fue accesible al 5,3\% de los docentes. La figura 5 recoge las causas que motivaron o justificaron la escasa realización de la vigilancia de la salud laboral. Observamos como la principal causa por la que este colectivo profesional manifestaba no haber realizado los exámenes de salud laboral durante el curso escolar fue la falta de información (46\%), o sea, casi la mitad el profesorado encuestado desconocía que la Consejería de Empleo está obligada a llevar a cabo la vigilancia de la salud laboral a través de los exámenes de salud. En segundo lugar, destaca el no ofertar dicha posibilidad (13\%) a los profesores. Es decir, sí sabían de su existencia, pero en su centro no se la ofertaron $(p<0,05)$. Subrayamos también que 17 profesionales mostraron desinterés por los exámenes de salud laboral (un 5\%), bien porque no los consideran necesarios o convenientes, bien porque se realizan sus propios reconocimientos o por otras razones (encontrarlos "repetitivos", "ineficaces" o por razones tan sorprendentes como que: "la directiva me dijo que no tenía derecho").

Figura 5. Causas de no realización de la vigilancia de la salud.

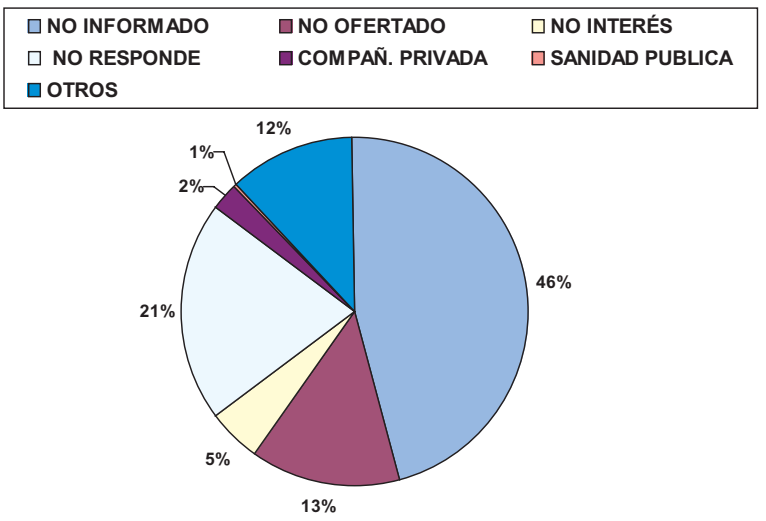




\section{DISCUSIONN}

El promedio de participación del profesorado encuestado fue del $44.25 \%$. Cifra que nos puede dar una idea del interés que muestran los docentes de la zona hacia el tema de la prevención de riesgos y la salud laboral. La proporción obtenida según género en la muestra del trabajo es muy parecida a la real de la población docente en Andalucía. Un estudio reciente (2) realizado por la misma Consejería de Educación indica que la proporción mujer/hombre actual es de $53 \%$ de mujeres frente al $47 \%$ de hombres.

Centrándonos en el apartado de salud laboral, obtenemos datos parecidos en cuanto a la patología más frecuente que existe en la población docente Andaluza (2). Destaca el predominio de la patología ORL e Infecciones, seguida de procesos osteomusculares y de alteraciones de salud mental. Especialmente, el que un $32 \%$ de los profesores/as encuestados/as (117 docentes) dicen padecer trastornos de la voz (afonías, disfonías...).

El tema de los trastornos de voz en los docentes es importante. Las enfermedades de la voz son citadas reiteradamente por la literatura relacionada con la salud laboral docente (8). Los problemas de la voz representan una de las patologías características que afectan, cada vez con más frecuencia, al personal docente. Numerosos estudios sitúan a la patología de la voz como uno de los diagnósticos más frecuentes en las bajas por enfermedad de los docentes $(3,5)$. La prevalencia que muestran diferentes estudios para los trastornos de la voz en los docentes varían desde, alrededor del $50 \%$ (9) hasta el $2.2 \%$ (Rodríguez y Gómez, 2001) pasando por el 12\% (10). En nuestro estudio ha sido del $32 \%$. Esta disparidad puede deberse a la diferente forma de codificar los trastornos de la voz (algunos lo hacen como problemas infecciosos, otros como otorrinolaringológicos, etc), por los servicios o asesorías médicas de las Delegaciones de Educación, que son las fuentes principales de los estudios previamente citados. Básicamente la mayoría se basan en la Clasificación Internacional de Enfermedades (CIE), aunque no siempre ocurre así. En todo caso, los expertos sobre el tema indican que las patologías orgánicas de la voz del profesorado triplican a las de otras profesiones (11). La prevalencia de la patología de la voz es también superior entre los docentes con relación a la población general (12). Recalca esta idea los resultados de un estudio descriptivo realizado en el personal laboral de centros educativos que excluía a los docentes: para una población de 1062 trabajadores no docentes, no se objetivó patología de la voz
(Gómez Armario, 1995). Además, la evolución en la incidencia de la patología de la voz se está incrementando considerablemente. Los datos estadísticos muestran un aumento extraordinariamente significativo de las enfermedades otorrinolaringológicas durante los últimos 20 años, con las consecuencias económicas que este hecho supone en lo que respecta a bajas por incapacidad laboral, sustituciones del personal, gastos sanitarios, etc. Y esto puesto que, junto con los trastornos mentales son las enfermedades con una Incapacidad Temporal de duración media más elevada con respecto a la media, manteniéndose como procesos cada vez más severos y crónicos que incrementan la duración de la incapacidad (12).

De ahí que desde distintos ámbitos se haya demandado desde hace tiempo, la inclusión de los trastornos de la voz en los docentes como enfermedad profesional. Hecho que ocurre desde la entrada en vigor el 1 de enero de 2007 del Real Decreto 1299/2006 (6). Éste incluye, por vez primera, una enfermedad profesional, dentro del grupo de enfermedades profesionales causadas por agentes físicos: en el anexo 1, código 2L0101 aparecen los "nódulos de las cuerdas vocales a causa de los esfuerzos sostenidos de la voz por motivos profesionales" para las "Actividades en las que se precise uso mantenido y continuo de la voz, como son los profesores, cantantes, actores, teleoperadores, locutores".

En el estudio que hemos realizado, 76 trabajadores/as (40\%) refirieron haber sido diagnosticados/as de alguna enfermedad o lesión relacionada con las cuerdas vocales. Dicha patología puede clasificarse dentro de las Disfonías funcionales con alteración estructural laríngea junto con otras (Edema de Reinke, pólipos laríngeos, granulomas...) producidas, generalmente en aquellas personas que realizan un abuso o mal uso de la voz y de la coordinación neumofónica; constituyendo la patología más frecuentes de la voz en el ámbito docente (12).

Por otra parte, llama atención que los encuestados en este trabajo apenas hayan citado trastornos como la fatiga ocular, o los relativos a la agudeza visual. Más si cabe cuando el protocolo de Pantallas de Visualización de Datos (PVD) puede aplicarse, junto con otros como el de "Posturas Forzadas"..., entre los existentes actualmente, para el profesorado.

Además, tengamos en cuenta que el RD 1299/2006 deja abierta la puerta, en su artículo 2, al reconocimiento de nuevas enfermedades en futuras modificaciones, recogiendo patologías cuya relación 
con el trabajo se sospecha aunque no esté suficientemente comprobada, lo que obliga a prestar una atención especial a dichas patologías y agentes, tanto físicas como psíquicas y psicosociales. $\mathrm{Al}$ respecto, algunos autores citan la interrelación entre patología psicosocial y los trastornos de la voz (13). El Real Decreto citado tiene también otras implicaciones en cuanto al mecanismo de iniciación de la notificación y comunicación de la enfermedad profesional (que afecta también a los facultativos del servicio de prevención), cambia el modelo de parte de ésta; e introduce un sistema de recogida de datos para su análisis epidemiológico por la unidad relativa al reconocimiento de los derechos derivados de enfermedad profesional (6).

La Ley de Prevención de Riesgos Laborales (7) establece que la Vigilancia de la Salud se realizará en función de los riesgos inherentes al puesto de trabajo y en base a unos protocolos. Por tanto, es necesaria (y preceptiva) la evaluación de riesgos previa de dicho puesto de trabajo. En relación con los protocolos de Vigilancia de la Salud, éstos deben tener en cuenta los factores de riesgo y las condiciones del medio de trabajo, y no deben realizarse de forma genérica. Cuando los reconocimientos médicos se hacen de forma general y homogénea, puede ocurrir que el trabajador disminuya su interés para realizarlos y por ende la frecuentación a la Unidad de Vigilancia de la Salud respectiva.

Por tanto, el examen médico de la vigilancia de la salud debería comenzar con la recogida de aquella información que tenga más relación con las patologías principales que afectan al puesto docente. Factores como la edad, el sexo, o el nivel de enseñanza influyen en la incidencia de la patología de la voz. De hecho, la patología nodular suele ser más frecuente en las mujeres que en los hombres, siendo más habitual en la tercera década de la vida (14). Estudios realizados en nuestro país (9) muestran que el perfil profesional de grupo de riesgo para padecer un trastorno de la fonación en el ámbito docente sería aquel de: "mujer joven, preferentemente maestra de infantil o primaria, especialmente para aquellas de idiomas, música o educación física, fumadora y/o expuesta al tabaco, la tiza o ambos, con antecedentes médicos de faringitis y síntomas como cansancio de la voz al finalizar la semana y cambios en el tono de voz, que hace un uso excesivo de la voz a nivel laboral, y con alteración en los parámetros acústicos". De ahí que propongamos preguntas sobre estas variables en el cuestionario.

Así mismo, es importante la semiología de datos como: el cansancio de la voz al finalizar la semana, los cambios en el tono de voz, la sensación de quemazón o el carraspeo.

Proponemos también recoger posibles hábitos perjudiciales. Por ejemplo, el abuso extralaboral de la voz al forzarla en ambientes ruidosos ("laringitis del fin de semana"), o hábitos nocivos como el tabaquismo, así como los tratamientos realizados previamente por el paciente. Es necesario incidir en programas de deshabituación tabáquica y prevenir el consumo de tabaco. La historia clínica debe incluir una exploración tanto física como instrumental. La exploración de la voz puede constituir una ocasión excelente para informar al paciente y realizar educación sanitaria, con el objeto de que comprenda mejor sus trastornos. Es a su vez conveniente comprender como "vive" el paciente su fonación. Algunas de las pruebas requieren la coordinación con otros profesionales sanitarios: especialista en ORL y/o foniatría, y logopedia básicamente. Planteamos este cuestionario como parte del examen de salud que puede realizar el especialista en medicina del trabajo ya que la evaluación acústica objetiva requiere pruebas específicas para la valoración funcional y son realizadas normalmente por el logopeda y el foniatra. Existen, no obstante, experiencias del uso de paquetes informáticos para analizar la voz en la Vigilancia de la Salud por el Médico del Trabajo, como es el caso de Navarra (15). Son también útiles para el diagnóstico las pruebas que permiten visualizar directa o indirectamente el aparato fonador (laringoscopia directa con monitor, estroboscopia, etc), permitiendo muchas de ellas el tratamiento informático de la información. Aunque estas pruebas de diagnóstico por la imagen puedieran ser realizadas por un especialista bien entrenado, el elevado coste de los aparatos y la necesidad de una formación específica hacen que sea poco realista su uso para la Vigilancia de la Salud. Algo parecido ocurre con la laringoscopia indirecta, cuyo coste es significativamente menor, pero que también requiere de un entrenamiento específico. Por tanto, una buena historia clínico-laboral con una anamnesis completa y una adecuada exploración que incluya también la rinoscopia y la otoscopia, junto con un buen diagnóstico diferencial, puede ser suficiente para establecer un diagnóstico de sospecha, que sea confirmado por el especialista en ORL y/o médico foniatra.

Aunque la misión principal de la Vigilancia de la Salud sea de tipo preventivo, recordemos la obligación de notificar adecuadamente la enfermedad profesional. Tanto para evitar la infradeclaración de estas enfermedades, como por las consecuencias de tipo económico y sanitario que implica para el pro- 
fesorado afectado. La Orden TAS/1/2007 establece el modelo del parte de enfermedad profesional y dicta las normas para su elaboración y transmisión (16). Además, en el personal funcionario, debe tenerse en cuenta la normativa pertinente (17).

La debida notificación no debe ir en detrimento de la acción preventiva que debería ser el principal objetivo. De ahí que en la secuencia propuesta de actuación incluyamos también la derivación a los servicios pertinentes para incluir a los grupos de más riesgo en acciones de tipo preventivo (formación, uso temporal de micrófonos...). Es fundamental para el profesorado la realización de ejercicios prácticos para el cuidado de la voz a modo de hábito rutinario en su trabajo $(18,19)$.

Para otros riesgos que conlleva la labor docente y en función de la evaluación de riesgos realizada, pueden aplicarse otros protocolos. Por ejemplo: el de posturas forzadas, Pantallas de Visualización de datos, o el de agentes biológicos en el caso de las Escuelas de Infantil. Es fundamental adaptarlos a las actividades que realice el profesorado en cuestión. Así, se tendrá en cuenta las posturas que adopta el profesorado de la educación infantil y primaria para atender al alumnado (flexión de tronco), los hábitos de trabajo (uso del ordenador, bipedestación prolongada...), y de salud (tomar bebidas frías, transporte en motocicleta para ir al trabajo...), las posturas inadecuadas (girar la cabeza hacia atrás mientras se escribe en la pizarra...), $\mathrm{u}$ otras circunstancias particulares, como puede ocurrir en el profesorado de educación física. En este sentido es fundamental que quien realice la Vigilancia de la Salud conozca la forma como trabaja el profesorado. La normativa establece que el examen de salud laboral se realizará con la información aportada tras la evaluación del puesto de trabajo por los técnicos competentes. Esta evaluación permitirá adoptar las medidas preventivas necesarias para prevenir futuras lesiones. Por citar un ejemplo, si se detecta una colocación inadecuada de la pizarra que lleva a la adopción posturas forzadas y éstas a trastornos músculoesqueléticos, habrá que corregirla mediante una intervención específica. 


\section{ANEXO I \\ CUESTIONARIO PARA EVALUAR LA FUNCIÓN FONATORIA \\ EN EL PROFESORADO}

\section{DATOS ADMINISTRATIVOS Y PROFESIONALES}

Apellidos y nombre:

Sexo:

Edad:

Documento de identificación sanitario:

CNAE:

CNO:

Doble presencia (realiza también el trabajo del hogar): sí / no

Centro de Trabajo:

Código:

Nivel de enseñanza que imparte:

\begin{tabular}{|c|c|c|c|c|c|}
\hline INFANTIL & PRIMARIA & SECUNDARIA & BACHILLER & $\begin{array}{c}\text { FORMACIÓN } \\
\text { PROFESIONAL }\end{array}$ & OTROS \\
\hline & & & & & \\
\hline
\end{tabular}

Número de años ejerciendo la docencia:

2. ANTECEDENTES LABORALES: Profesiones previas y riesgos asociados

- ACCIDENTES DE TRABAJO:

\begin{tabular}{|l|l|l|l|}
\hline FECHA & LESIÓN & CAUSA & BAJA \\
\hline & & & \\
\hline
\end{tabular}

- BAJAS LABORALES

3. DESCRIPCION DEL PUESTO DE TRABAJO: Actividades que realiza, lugar o lugares de trabajo donde lleva a cabo la mayor parte de su jornada laboral (aula, gimnasio, taller, laboratorio...), posición predominante que adopta durante el desarrollo de las clases (de pie, sentado), tiempo de trabajo, uso de ordenador y duración del mismo. 
- Hábitos de trabajo: ¿gira la cabeza hacia atrás mientras escribe en la pizarra? ¿Realiza un "calentamiento de la voz" antes de comenzar la clase?...

- Evaluación de riesgos del puesto de trabajo:

- Fecha de realización:

- Técnico responsable:

- Riesgos detectados:

- Medidas preventivas adoptadas:

4. ANTECEDENTES MÉDICOS FAMILIARES (orientada fundamentalmente hacia patología orgánica y funcional de la voz):

\section{ANTECEDENTES MÉDICOS PERSONALES:}

- Laringitis: $\quad$ Faringitis: Disfonías:

- Nódulos de cuerdas vocales:

- Otra afectación de las cuerdas vocales (pólipos, granulomas...)

- Otros problemas de la voz:

- Reflujo gastro esofágico:

- Problemas ginecológicos:

- Procesos cancerosos:

- Otras patologías:

6. ¿TRABAJADOR ESPECIALMENTE SENSIBLE? (embarazadas, discapacitados, patologías previas...)

7. ALERGIAS: No / Sí (Especificarlas) 


\section{TRATAMIENTOS:}

\section{ANAMNESIS CENTRADA EN EL APARATO FONADOR}

\section{HABITOS PERSONALES}

- Fuma:

\begin{tabular}{|l|l|l|l|}
\hline Muy a menudo & A veces & Raramente & Nunca \\
\hline & & & \\
\hline
\end{tabular}

- Consume alcohol:

\begin{tabular}{|l|l|l|l|}
\hline Muy a menudo & A veces & Raramente & Nunca \\
\hline & & & \\
\hline
\end{tabular}

- Realiza una alimentación equilibrada:

\begin{tabular}{|l|l|l|l|}
\hline Muy a menudo & A veces & Raramente & Nunca \\
\hline & & & \\
\hline
\end{tabular}

- Practica ejercicio físico

\begin{tabular}{|l|l|l|l|}
\hline Muy a menudo & A veces & Raramente & Nunca \\
\hline & & & \\
\hline
\end{tabular}

- Fuerza la voz durante el fin de semana

\begin{tabular}{|l|l|l|l|}
\hline Muy a menudo & A veces & Raramente & Nunca \\
\hline & & & \\
\hline
\end{tabular}

- Nota ardor o quemazón que le sube desde el estómago

\begin{tabular}{|l|l|l|l|}
\hline Muy a menudo & A veces & Raramente & Nunca \\
\hline & & & \\
\hline
\end{tabular}
DUERME: < 6 horas:
Entre 6 y 8 horas: $\quad$ Más de 8 horas:

Considera su sueño:

Reparador:

No reparador: 


\section{DURANTE SU TRABAJO}

1. Pierde la voz habitualmente sí no

2. Nota cambios en el tono de voz sí no

3. Nota sensación de cuerpo extraño al tragar sí no

4. Realiza un uso excesivo de la voz sí no

5. Existe ruido en el ambiente que le dificulta el habla sí no

6. Le cuesta respirar sí no

7. Tiene dificultades para relajarse

sí no

\section{DURANTE O DESPUÉS DEL TRABAJO, SIENTE USTED:}

1. Picor, ardor o irritación en la garganta

\begin{tabular}{|l|l|l|l|}
\hline Muy a menudo & A veces & Raramente & Nunca \\
\hline & & & \\
\hline
\end{tabular}

2. Dolores en la garganta

\begin{tabular}{|l|l|l|l|}
\hline Muy a menudo & A veces & Raramente & Nunca \\
\hline & & & \\
\hline
\end{tabular}

3. Carraspeo.

\begin{tabular}{|l|l|l|l|}
\hline Muy a menudo & A veces & Raramente & Nunca \\
\hline & & & \\
\hline
\end{tabular}

4. Una fatiga al hablar prematura a la hora de dar las clases

\begin{tabular}{|l|l|l|l|}
\hline Muy a menudo & A veces & Raramente & Nunca \\
\hline & & & \\
\hline
\end{tabular}

5. Nota usted cansancio en su voz a medida que se acerca el fin de semana

\begin{tabular}{|l|l|l|l|}
\hline Muy a menudo & A veces & Raramente & Nunca \\
\hline & & & \\
\hline
\end{tabular}


6. Modifica usted habitualmente su tono de voz para hacerse oír (yendo a tonos más graves)

\begin{tabular}{|l|l|l|l|}
\hline Muy a menudo & A veces & Raramente & Nunca \\
\hline & & & \\
\hline
\end{tabular}

7. Si noto alguno de estos síntomas, mejoran:

\begin{tabular}{|l|l|l|}
\hline & SÍ & NO \\
\hline Tras el fin de semana & & \\
\hline Después de las vacaciones & & \\
\hline
\end{tabular}

8. Describa, si las tiene, otras sensaciones en relación con el uso de la voz:

OTRAS CIRCUNSTANCIAS: ¿Suele abrigarse el cuello en épocas de frío? ¿Coincide las molestias en la voz con la menstruación? ¿Acude al trabajo en motocicleta?...

10. EXPLORACIÓN FÍSICA (Detallar los hallazgos principales)

INSPECCIÓN: general y de la cavidad bucal.

PALPACIÓN: buscando adenopatías submandibulares, retroauriculares, supra e inflraclaviculares, axilares...

AUSCULTACIÓN: cardiopulmonar

OTOSCOPIA:

\section{EXPLORACIÓN COMPLEMENTARIA}

Un estudio realizado por la Sección de Prevención de Riesgos Laborales del Departamento de Educación del Gobierno de Navarra (Esandi et al, 2007) concluye que es aconsejable incluir el análisis funcional de la voz en el protocolo de Vigilancia de Salud específica en docentes como herramienta preventiva para el diagnóstico de patología funcional de la voz. Estas autoras indican que puede utilizarse como instrumento la aplicación "Voice Assesment" del programa Dr. Speech 3.0 u otros similares. Este análisis debe complementarse, según indican los autores, con la exploración laríngea realizada por el facultativo especialista en Otorrino Laringología (ORL) / foníatra para el diagnóstico de patología orgánica, así como para el diagnóstico de confirmación de la patología funcional. 


\section{DIAGNÓSTICO DE SOSPECHA DE NÓDULOS DE CUERDAS VOCALES: SÍ / NO}

Si se realiza el diagnóstico de confirmación por el especialista, el médico del Servicio de Prevención o del Sistema Nacional de Salud debe tramitarlo al INSS para calificar la contingencia como enfermedad profesional según establece la legislación vigente.

Insistimos en que lo ideal sería haber detectado precozmente las primeras molestias (concepto de "vigilancia"), adoptado e implementado las medidas necesarias en las primeras etapas de la enfermedad, tanto en el individuo (formación, información, actuaciones logopédicas y foniátricas), como en la mejora de las condiciones de trabajo (condiciones acústicas del espacio de trabajo, disminución del ruido, disciplina en el aula, mobiliario adecuado, medidas organizativas, etc).

A continuación se propone un CRONOGRAMA DE ACTUACIÓN orientativo:






\section{BIBLIOGRAFIA}

1. Prevención de Riesgos Laborales. 1999. Madrid. Las Enfermedades Profesionales. Madrid: Ministerio de Educación Cultura y Deporte; 1999.

2. ACUERDO de 19 de septiembre de 2006, del Consejo de Gobierno, por el que se aprueba el I Plan Andaluz de Salud Laboral y Prevención de Riesgos Laborales del personal docente de los centros públicos dependientes de la Consejería de Educación (2006-2010)". BOJA núm. 196 de 9 de octubre de 2006. p:12

3. García Calleja M. Enfermedades del profesorado: análisis y prevención. Cuad Pedag 1989; 192: 67-72.

4. Esteve JM, Franco S, Vera J. Los profesores ante el cambio social: repercusiones sobre la evolución de la salud de los profesores. 1995. Anthropos; 1995.

5. Sevilla U, Villanueva R. La salud laboral docente en la enseñanza pública. 2000. Madrid. Federación de enseñanza. CCOO; 2000.

6. REAL DECRETO 1299/2006, de 10 de noviembre, por el que se aprueba el cuadro de enfermedades profesionales en el sistema de la Seguridad Social y se establecen criterios para su notificación y registro. BOE núm. 302 de 19 de diciembre de 2006. pp: 44487-44546.

7. LEY $31 / 1995$ de 8 de noviembre, de Prevención de Riesgos Laborales. BOE núm. 269. de 10 de noviembre de 1995. Modificada por la Ley 54/2003, de 12 de diciembre, de reforma del marco normativo de la prevención de riesgos laborales.

8. Ruiz J, Campoy TJ, Cañas A (coord.). Estudio sobre la salud en la enseñanza no universitaria. 1996. Universidad de Jaén; 1996.

9. Cortazar MI, Kareaga G, Lansac M, Hirsuta JA, Azuara S. Estudio de los trastornos de voz en docentes y auxiliares de educación especial de la enseñanza pública de Bizkaia. Servicio Médico de la Delegación de Educación de Bizkaia 2002; Gobierno Vasco.

10. García Calleja M. Prevención de riesgos de la voz en docentes. Rev Univ Cienc Trab; 1997; 1: 199-223.

11. Gañet RE, Martínez MP. La voz: instrumento de trabajo y factor de riesgo laboral. Med Segur Trab 2003; 192: $35-46$

12. López-Tappero J. Disfonías profesionales. Rev Español Foniatr 1988; 1 (2):36-42
13. Chavkravorty B. Mental health among school teachers. In M.Cole y S.Walker (Eds.), Teaching and Stress. 1989. Philadelphia. Open University Press; 1986.

14. Le Huche F, Allai A. La voz. Patología vocal: semiología y disfonías disfuncionales. Barcelona. 1994. Editorial Masson; 1994.

15. Esandi A, Cilveti S, Abárzuza JJ, Sagasti MA, Urricelqui A, Colomo A. Valoración objetiva de las alteraciones de la voz del profesorado mediante análisis acústico vocal. Póster presentado en el XVI Congreso de la Sociedad Española de Salud Laboral en la Administración Pública. Pamplona, marzo 2007.

16. ORDEN TAS/1/2007, de 2 de enero, por la que se establece el modelo de parte de enfermedad profesional, dicta normas para su elaboración y transmisión y crea el correspondiente fichero de datos personales. BOE núm. 4 de 4 de enero de 2005. 482-87

17. ORDEN APU/3554/2005, de 7 de noviembre, por la que se regula el procedimiento para el reconocimiento de los derechos derivados de enfermedad profesional y de accidente en acto de servicio en el ámbito del mutualismo administrativo gestionado por MUFACE. BOE núm. 275 de 17 de noviembre de 2005. pp: 37572-37580

18. Quiñones C. El cuidado de la voz. Ejercicios prácticos. 1997. Madrid. Editorial Escuela Española; 1997.

19. Cortázar MI, Rojo B. La voz en la docencia. Conocer y cuidar nuestra herramienta de trabajo, 2007. Graó

\section{Obras no publicadas:}

Gómez Armario I. Estudio epidemiológico de las incapacidades laborales temporales del personal no docente de la Delegación Provincial de Educación de Cádiz. Curso escolar 1994-1995, Boletín epidemiológico. Servicio Andaluz de Salud, 1995; 54. Cádiz.

Rodríguez MC, Gómez Armario I. Estudio de las incapacidades en el profesorado no universitario de la provincia de Cádiz, 2001. Servicio médico de la Delegación Provincial de Educación de Cádiz. Consejería de Educación y Ciencia. Junta de Andalucía. 